\title{
Sticky Particle Dynamics on the Real Line
}
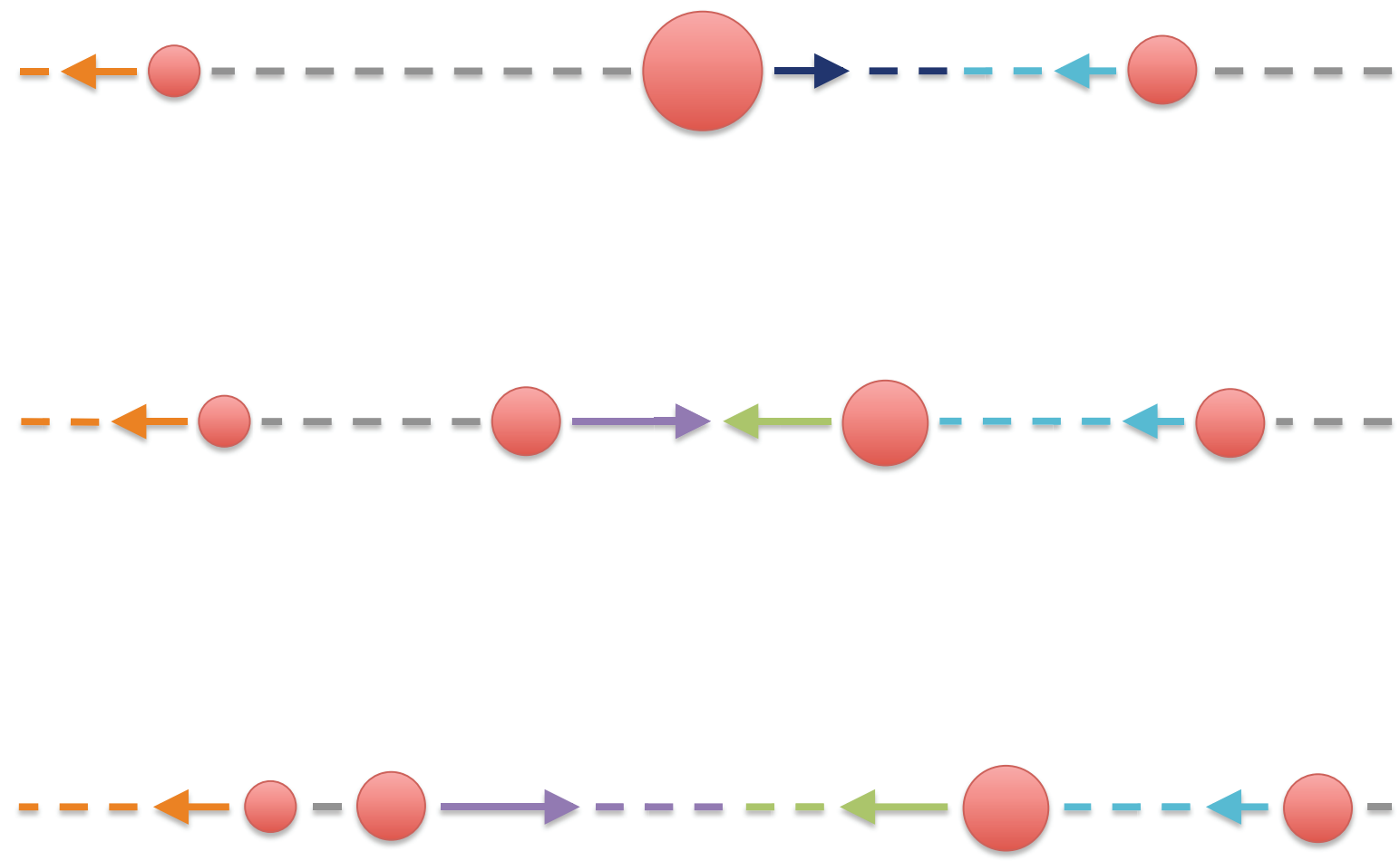

\section{Ryan Hynd}

\section{Introduction}

At the simplest level, the physics of adhesion can be modeled with perfectly inelastic collisions. Let us recall this notion by considering the example of two point masses in $\mathbb{R}$ that each move with constant velocity until they collide. To fix ideas, we assume these particles have respective masses $m_{1}, m_{2}>0$ and velocities $v_{1}, v_{2} \in \mathbb{R}$. Upon colliding, these particles undergo a perfectly inelastic collision provided they merge to form a single particle of mass $m_{1}+m_{2}$ and the resulting particle travels with velocity $v$

Ryan Hynd is an associate professor of mathematics at the University of Pennsylvania. He is partially supported by NSF grant DMS-1554130. His email address is rhynd@math. upenn. edu.

Communicated by Notices Associate Editor Notices Associate Editor Daniela De Silva.

For permission to reprint this article, please contact:

reprint-permission@ams.org.

DOI: https://doi.org/10.1090/noti/1788 chosen to satisfy

$$
m_{1} v_{1}+m_{2} v_{2}=\left(m_{1}+m_{2}\right) v
$$

Refer to Figure 1. Note in particular that mass and momentum are conserved during the collision. Moreover, this example is easily generalized to any number of colliding particles.

It turns out that the elementary mechanics of perfectly inelastic collisions can be used to model the evolution of matter that is not subject to pressure. This observation is usually attributed to Zel'dovich in his work on the early stages of galaxy formation [18]. His theory was further developed by other cosmologists who studied the role of adhesion in the dynamics of large-scale structures in the universe $[7,10,15,16]$.

In this article, we will discuss some of the mathematics involved in Zel'dovich's theory. A system of equations which embodies the conservation of mass and momentum 


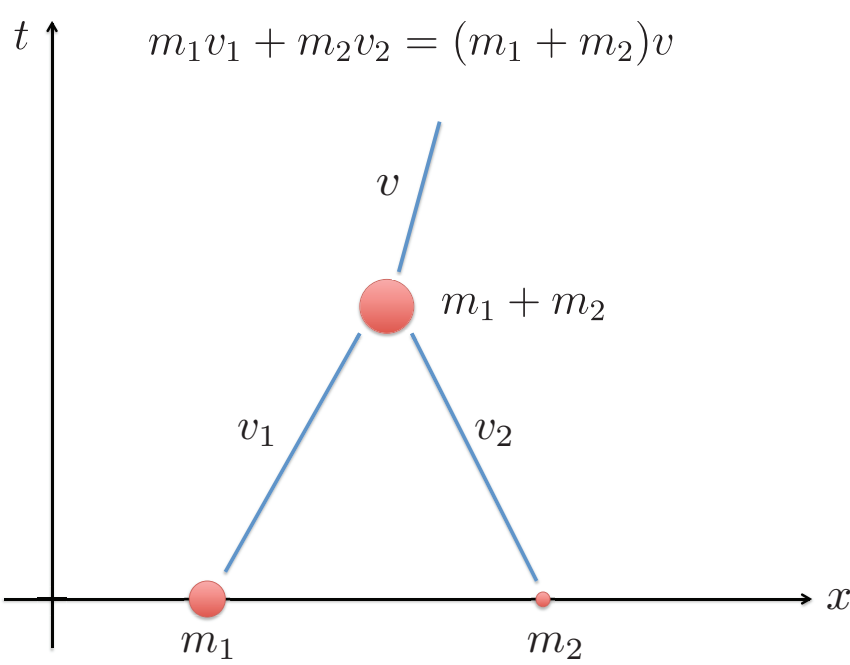

Figure 1. A perfectly inelastic collision between two point masses in $\mathbb{R}$. Here $x$ denotes a variable on the real line and $t$ denotes time. The particles are sketched to be larger than points in order to highlight the possibility that they may have different masses. Further, the particle resulting from the collision is sketched even larger to highlight that it is more massive.

of interacting particles will play an important role. This system also describes how to best move from one probability distribution to another in the theory of optimal mass transportation. First, we will build on our example above and examine finite collections of point masses on the real line that interact only via perfectly inelastic collisions.

\section{Finitely Many Interacting Particles}

Let us consider $N$ particles on the real line that have masses $m_{1}, \ldots, m_{N} \geq 0$. We suppose $m_{1}+\cdots+m_{N}=1$ and that these point masses move freely unless they collide. When any sub-collection of these particles collide, they will undergo a perfectly inelastic collision. We will write $\gamma_{1}, \ldots, \gamma_{N}:[0, \infty) \rightarrow \mathbb{R}$ for the trajectories that are associated with the respective masses $m_{1}, \ldots, m_{N}$. That is, $\gamma_{i}(t)$ is the location of point mass $m_{i}$ at time $t \geq 0$, which could be by itself or part of a larger mass if it has collided with other particles prior to time $t$. A schematic of the graphs of $\gamma_{1}, \ldots, \gamma_{N}$ is displayed in Figure 2 .

These sticky particle trajectories are continuous and piecewise linear, and they have two important properties. The first is that they satisfy the inequality:

$$
\frac{1}{t}\left|\gamma_{i}(t)-\gamma_{j}(t)\right| \leq \frac{1}{s}\left|\gamma_{i}(s)-\gamma_{j}(s)\right|
$$

for all $i, j=1, \ldots, N$ and $0<s \leq t$. Observe that this inequality quantifies the fact that if two particles collide at time $S$, they will remain stuck together for all times $t \geq s$.

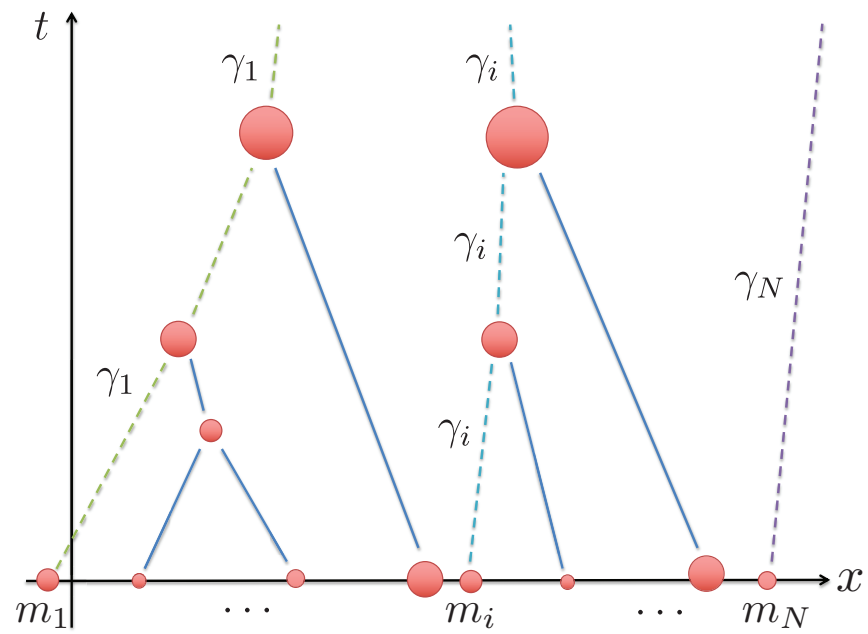

Figure 2. Sticky particle trajectories $\gamma_{1}, \ldots, \gamma_{N}$ in $\mathbb{R} \times(0, \infty)$ that track $N$ particles moving freely in $\mathbb{R}$ that only interact via perfectly inelastic collisions. The corresponding masses $m_{1}, \ldots, m_{N}$ are drawn with different sizes to indicate that they are not assumed to be identical. The three dashed trajectories $\gamma_{1}, \gamma_{i}$, and $\gamma_{N}$ emphasize that these trajectories track $m_{1}, m_{i}$, and $m_{N}$, respectively.

The second feature is

$$
\sum_{\gamma_{i}(t)=\gamma_{j}(t)} m_{i} \dot{\gamma}_{i}(t-)=\left(\sum_{\gamma_{i}(t)=\gamma_{j}(t)} m_{i}\right) \dot{\gamma}_{j}(t+)
$$

for $j=1, \ldots, N$ and $t>0$. Here $\dot{\gamma}_{i}(t-)$ and $\dot{\gamma}_{i}(t+)$ denote the limits from the left and right of the slope of $\gamma_{i}$ at $t$, respectively; and each summation is over $i=1, \ldots, N$ for which $\gamma_{i}(t)=\gamma_{j}(t)$. We note that this identity encodes the conservation of momentum that occurs in between and during collisions. Both properties are discussed in section 3 of [9].

Since the total mass of this physical system is conserved, it makes sense for us to consider the space $\mathcal{P}(\mathbb{R})$ of (Borel) probability measures on $\mathbb{R}$. We can then regard the mass distribution of particles as the map $\rho:[0, \infty) \rightarrow \mathcal{P}(\mathbb{R}) ; t \mapsto$ $\rho_{t}$, where

$$
\rho_{t}:=\sum_{i=1}^{N} m_{i} \delta_{\gamma_{i}(t)} .
$$

Here $\delta_{z}$ is the Dirac measure at $z \in \mathbb{R}$. That is,

$$
\delta_{z}(A)= \begin{cases}1, & z \in A \\ 0, & z \notin A\end{cases}
$$

for $A \subset \mathbb{R}$. As a result, $\rho_{t}(A)$ is the fraction of mass within $A$ at time $t$. A corresponding velocity is a (Borel) function $v: \mathbb{R} \times[0, \infty) \rightarrow \mathbb{R}$ for which

$$
v(x, t)=\dot{\gamma}_{i}(t+) \text { when } x=\gamma_{i}(t) .
$$




\section{The Sticky Particle System (SPS)}

As it turns out, the mass distribution $\rho$ defined in (3) and velocity $v$ specified in (4) satisfy

$$
\int_{0}^{\infty} \int_{\mathbb{R}}\left(\partial_{t} \phi+v \partial_{x} \phi\right) d \rho_{t} d t+\int_{\mathbb{R}} \phi(\cdot, 0) d \rho_{0}=0
$$

and

$$
\int_{0}^{\infty} \int_{\mathbb{R}}\left(\partial_{t} \phi+v \partial_{x} \phi\right) v d \rho_{t} d t+\int_{\mathbb{R}} \phi(\cdot, 0) v_{0} d \rho_{0}=0
$$

for each smooth $\phi: \mathbb{R} \times[0, \infty) \rightarrow \mathbb{R}$ with compact support. Here

$$
\rho_{0}=\sum_{i=1}^{N} m_{i} \delta_{\gamma_{i}(0)}
$$

is the initial mass distribution and $v_{0}: \mathbb{R} \rightarrow \mathbb{R}$ is any function such that $v_{0}\left(\gamma_{i}(0)\right)=\dot{\gamma}_{i}(0)$ for $i=1, \ldots, N$. We note that the averaging property (2) is an important ingredient in the derivation of (6).

We interpret (5) and (6) to mean that the pair $\rho$ and $v$ is a solution of the conservation of mass equation

$$
\partial_{t} \rho+\partial_{x}(\rho v)=0
$$

and the conservation of momentum equation

$$
\partial_{t}(\rho v)+\partial_{x}\left(\rho v^{2}\right)=0
$$

in $\mathbb{R} \times(0, \infty)$, respectively, that satisfies the initial conditions

$$
\left.\rho\right|_{t=0}=\rho_{0} \quad \text { and }\left.\quad \nu\right|_{t=0}=v_{0} .
$$

The partial differential equations (8) and (9) together are known as the sticky particle system (SPS), and they describe the dynamics of one dimensional matter that is not subject to pressure. Our notion of solution is consistent in the sense that if the pair $\rho$ and $\nu$ were a conventional solution, we could multiply the equations (8) and (9) by $\phi$ and integrate by parts to derive (5) and (6), respectively. We will also say that $\rho$ and $v$ is a solution pair of the SPS on $\mathbb{R} \times(0, \tau)$ with initial conditions (10) provided (5) and (6) hold for all $\phi$ that are supported in $\mathbb{R} \times[0, \tau)$.

We now know that we can always find a solution of the SPS when the initial mass density arises from a finite collection of point masses and is specifically of the form (7). We would like to extend these considerations to general initial mass distributions $\rho_{0} \in \mathcal{P}(\mathbb{R})$. Our goal is to prescribe the mass distribution and velocity at time 0 and then use the SPS to describe the evolution of the mass distribution and velocity at all later times. We will make a few observations before further exploring this fundamental existence question.

\section{The Method of Characteristics}

The SPS always has a solution for a given initial mass distribution $\rho_{0} \in \mathcal{P}(\mathbb{R})$ provided that the initial velocity $v_{0}$ is continuous and nondecreasing. In this case, the trajectories $t \mapsto x+t v_{0}(x)$ and $t \mapsto y+t v_{0}(y)$ do not coincide for distinct $x, y \in \mathbb{R}$ since the function $\mathrm{id}_{\mathbb{R}}+t v_{0}$ is increasing. As a result, the initial mass distribution is simply translated along linear trajectories that do not intersect. With this observation, we define $\rho_{t}$ by

$$
\int_{\mathbb{R}} g(y) d \rho_{t}(y):=\int_{\mathbb{R}} g\left(x+t v_{0}(x)\right) d \rho_{0}(x)
$$

for each $g$ belonging to the space $C_{b}(\mathbb{R})$ of bounded continuous functions on $\mathbb{R}$. This definition can be written more concisely as

$$
\rho_{t}:=\left(\operatorname{id}_{\mathbb{R}}+t v_{0}\right)_{\#} \rho_{0}, \quad t \geq 0 .
$$

Since the trajectories are linear, any velocity $v: \mathbb{R} \times$ $[0, \infty) \rightarrow \mathbb{R}$ associated with $\rho$ must satisfy

$$
v\left(x+t v_{0}(x), t\right)=v_{0}(x), \quad x \in \mathbb{R}, t \in[0, \infty)
$$

as shown in Figure 3. That is,

$$
v(\cdot, t):=v_{0} \circ\left(\mathrm{id}_{\mathbb{R}}+t v_{0}\right)^{-1} .
$$

We leave it as an exercise to check that the pair $\rho$ and $v$ is indeed a solution of SPS with initial conditions $\rho_{0}$ and $v_{0}$.

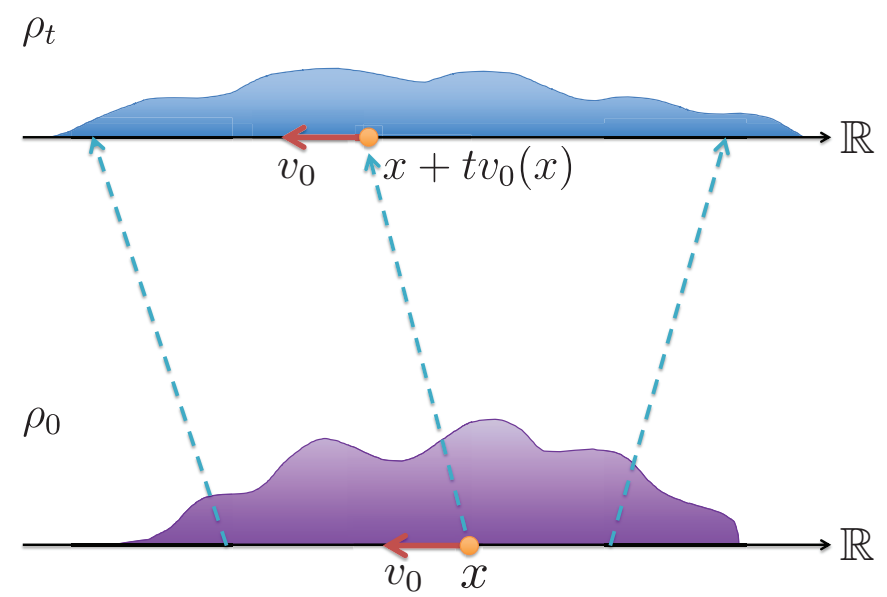

Figure 3. The evolution of the mass $\rho_{0}$ with nondecreasing initial velocity $v_{0}$ at time $t \geq 0$. The purple shape is a schematic of the mass distribution of $\rho_{0}$; the blue shape is a schematic of the evolved mass $\rho_{t}$; the dashed lines represent the linear trajectories which do not cross. The initial velocity of the particle starting at $x$ is $v_{0}(x)$; its velocity $v$ at position $x+t v_{0}(x)$ and time $t$ is also equal to $v_{0}(x)$.

When $v_{0}$ isn't nondecreasing, we can sometimes build on this example to obtain a solution of the SPS on $\mathbb{R} \times$ $(0, \boldsymbol{\tau})$ for some $\boldsymbol{\tau}>0$ and sufficiently small. In particular, 
if id $_{\mathbb{R}}+t v_{0}$ is increasing for each $t \in[0, \tau), \rho$ defined in (11) and $v$ specified in (12) is a solution pair of the SPS on $\mathbb{R} \times(0, \tau)$ with initial conditions $\rho_{0}$ and $\nu_{0}$.

\section{Optimal Mass Transportation on the Real Line}

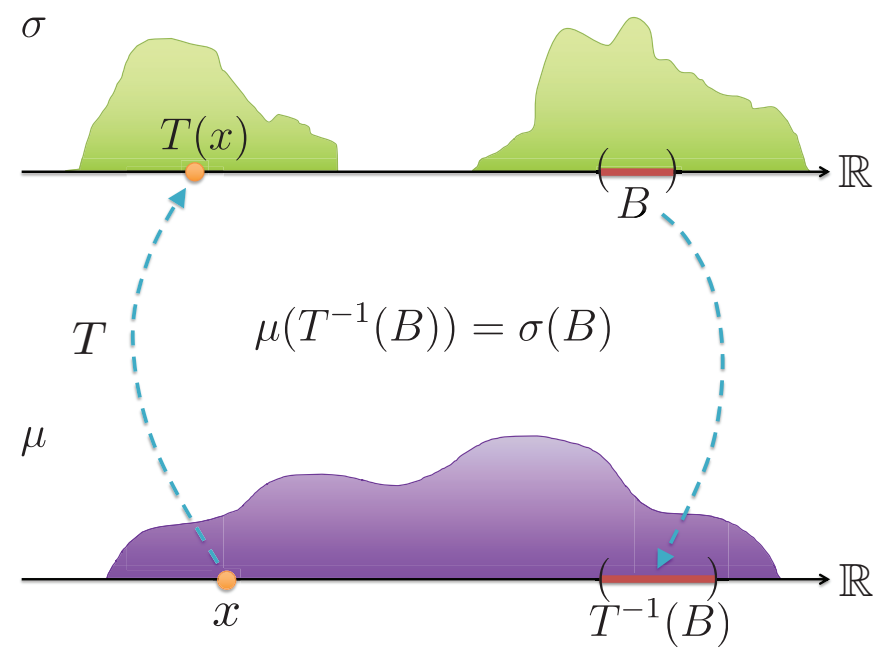

Figure 4. A function $T$ that transports mass in a measure preserving way from a distribution $\mu$ (in purple) which represents a pile of sand to another distribution $\sigma$ (in green) representing a hole to be filled.

In a 1781 memoir [12], Monge famously asked: how do you best move a given pile of sand to fill up a given hole of the same total volume? We will model the sand and hole as measures belonging to

$$
\mathcal{P}_{2}(\mathbb{R}):=\left\{\mu \in \mathcal{P}(\mathbb{R}): \int_{\mathbb{R}} x^{2} d \mu(x)<\infty\right\}
$$

and consider the following version of Monge's problem on the real line. For given $\mu, \sigma \in \mathcal{P}_{2}(\mathbb{R})$, find $T: \mathbb{R} \rightarrow \mathbb{R}$ that minimizes the total cost of transportation

$$
\int_{\mathbb{R}}(x-T(x))^{2} d \mu(x)
$$

among all $T$ satisfying the measure preserving constraint $T_{\#} \mu=\sigma$. This means

$$
\int_{\mathbb{R}} g(y) d \sigma(y)=\int_{\mathbb{R}} g(T(x)) d \mu(x)
$$

for each $g \in C_{b}(\mathbb{R})$ or equivalently that $\sigma(B)=\mu\left(T^{-1}(B)\right)$ for each $B \subset \mathbb{R}$. Refer to Figure 4 .

It turns out that if $\mu$ does not charge mass to points, our version of Monge's problem has a solution given by a nondecreasing function $T^{*}: \mathbb{R} \rightarrow \mathbb{R}$. In particular, this function is known as the monotone rearrangement of $\mu$ onto $\sigma$ and it can be written rather explicitly in terms of the distribution functions of $\mu$ and $\sigma$ (as detailed in section 2.2 of [17]). Using this optimal $T^{*}$, McCann introduced the displacement interpolation between $\mu$ and $\sigma$ as

$$
\rho_{t}=\left((1-t) \operatorname{id}_{\mathbb{R}}+t T^{*}\right)_{\#} \mu
$$

for $0 \leq t \leq 1$ [11]. Note that $\rho_{0}=\mu, \rho_{1}=\sigma$, and $(1-t) \mathrm{id}_{\mathbb{R}}+t T^{*}=\mathrm{id}_{\mathbb{R}}+t\left(T^{*}-\mathrm{id}_{\mathbb{R}}\right)$ is an increasing function for $0 \leq t<1$.

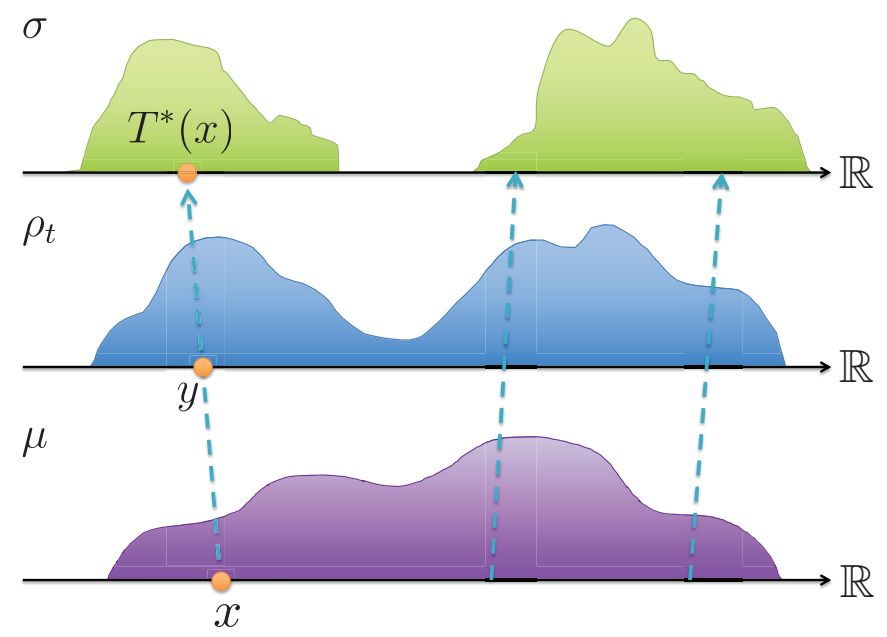

Figure 5. The optimal transportation function $T^{*}$ that rearranges $\mu$ onto $\sigma$ shown with the displacement interpolation $\rho_{t}$ between $\mu$ and $\sigma$ for some $t \in[0,1]$. Here $y=(1-t) x+t T^{*}(x)$, and we emphasize that mass is optimally transported along the linear trajectories between the support of $\mu$ and the support of $\sigma$.

If $T^{*}$ is additionally continuous, we can set

$$
v(\cdot, t)=\left(T^{*}-\mathrm{id}_{\mathbb{R}}\right) \circ\left((1-t) \mathrm{id}_{\mathbb{R}}+t T^{*}\right)^{-1}
$$

for $t \in[0,1)$. In view of (11) and (12), the pair $\rho: t \mapsto$ $\rho_{t}$ and $v$ is a solution of the SPS on $\mathbb{R} \times(0,1)$ with initial conditions $\rho_{0}=\mu$ and $v_{0}=T^{*}-\mathrm{id}_{\mathbb{R}}$. This solution corresponds to intersection-less trajectories and it means that mass is optimally transferred from the support of $\mu$ to the support of $\sigma$ along straight lines. See Figure 5.

The square root of the minimal cost in Monge's problem is related to a quantity known as the Wasserstein distance

$$
W_{2}(\mu, \sigma):=\inf _{\pi}\left(\int_{\mathbb{R} \times \mathbb{R}}(x-y)^{2} d \pi(x, y)\right)^{1 / 2}
$$

between $\mu$ and $\sigma$. Here the infimum is taken over (Borel) probability measures $\pi$ on $\mathbb{R} \times \mathbb{R}$ satisfying

$$
\pi(A \times \mathbb{R})=\mu(A) \text { and } \pi(\mathbb{R} \times A)=\sigma(A)
$$

for each $A \subset \mathbb{R}$. We note that the Wasserstein space $\left(\mathcal{P}_{2}(\mathbb{R}), W_{2}\right)$ is a complete, separable metric space.

In the case that $\mu$ does not charge mass to points, the displacement interpolation $\rho$ between $\mu$ and $\sigma$ satisfies

$$
W_{2}\left(\rho_{t}, \rho_{s}\right)=(t-s) W_{2}(\mu, \sigma), \quad 0 \leq s \leq t \leq 1 .
$$


As a result, $\rho$ is a constant speed geodesic. These ideas extend to general $\mu \in \mathcal{P}(\mathbb{R})$ which may charge mass to points. Indeed, the Benamou-Brenier formula [1] and the computations performed on action minimizing paths in the Wasserstein space by Gangbo, Nguyen, and Tudorascu [6] together imply that all constant speed geodesics in the Wasserstein space correspond to solutions of the SPS.

\section{Solution for Given Initial Conditions}

Now let us return to the initial value problem for the SPS, which is posed as follows.

Initial value problem: For a given $\rho_{0} \in \mathcal{P}(\mathbb{R})$ and $\nu_{0} \in$ $C_{b}(\mathbb{R})$, find $\rho:[0, \infty) \rightarrow \mathcal{P}(\mathbb{R})$ and $v: \mathbb{R} \times[0, \infty) \rightarrow \mathbb{R}$ that satisfy (5) and (6).

The existence of a solution to this initial value problem was first established by Weinan, Rykov, and Sinai [5] and by Brenier and Grenier [2] using novel methods based on Hamilton-Jacobi equations and conservations laws, respectively. Natile and Savaré [13] subsequently extended and merged both of these approaches by employing a flow in Lagrangian variables. We also mention that Dermoune [4] solved this problem using probabilistic techniques.

All of these approaches rely on the fact that convex combinations of Dirac measures are dense in $\mathcal{P}(\mathbb{R})$. That is, for a given $\rho_{0} \in \mathcal{P}(\mathbb{R})$, there is a sequence $\left(\rho_{0}^{k}\right)_{k \in \mathbb{N}}$ such that each $\rho_{0}^{k}$ is of the form (7) and

$$
\int_{\mathbb{R}} g(x) d \rho_{0}(x)=\lim _{k \rightarrow \infty} \int_{\mathbb{R}} g(x) d \rho_{0}^{k}(x)
$$

for each $g \in C_{b}(\mathbb{R})$. Recall that when the initial mass distribution is of the form (7), we can solve the initial value problem using sticky particle trajectories. Therefore, for a given initial velocity $v_{0} \in C_{b}(\mathbb{R})$ and $k \in \mathbb{N}$, we can find a solution pair $\rho^{k}$ and $v^{k}$ of the SPS with initial conditions

$$
\left.\rho^{k}\right|_{t=0}=\rho_{0}^{k} \text { and }\left.\quad v^{k}\right|_{t=0}=v_{0} \text {. }
$$

It turns out that we can send $k \rightarrow \infty$ along a subsequence to obtain a solution pair $\rho$ and $v$ of the SPS with the desired initial mass distribution $\rho_{0}$ and velocity $v_{0}$ [9]. The two key features which allow for this type of compactness are as follows. The first is

$$
\left(v^{k}(x, t)-v^{k}(y, t)\right)(x-y) \leq \frac{1}{t}(x-y)^{2}
$$

for each $t>0$ and $x, y$ in the support of $\rho_{t}^{k}$; this inequality follows directly from (1). The second feature is

$$
\int_{\mathbb{R}} \frac{1}{2}\left(v^{k}(x, t)\right)^{2} d \rho_{t}^{k}(x) \leq \int_{\mathbb{R}} \frac{1}{2}\left(v_{0}(x)\right)^{2} d \rho_{0}^{k}(x)
$$

for $t \geq 0$, which can be derived from (2) using Jensen's inequality. This solution pair $\rho$ and $v$ will also satisfy a version of inequality (13), and Huang and Wang showed that it is the unique solution pair of the SPS for given initial conditions with this property [8].

\section{Instability in Higher Dimensions}

Many of the ideas that we have discussed so far apply to sticky particle dynamics in $\mathbb{R}^{d}$. However, there is a fundamental difference between the associated dynamics when $d=1$ and when $d>1$. To see this disparity, let us consider a perfectly inelastic collision of two point masses in $\mathbb{R}^{2}$. We will suppose these particles have masses $m_{1}, m_{2}>$ 0 and move with constant velocity $v_{1}, v_{2} \in \mathbb{R}^{2}$, respectively. When they collide, they form a single particle of mass $m_{1}+m_{2}$ that has constant velocity $v$ given by

$$
m_{1} v_{1}+m_{2} v_{2}=\left(m_{1}+m_{2}\right) v
$$

as shown in Figure 6a.

Let's further assume that these particles are not restricted to move on the same line. In this case, we can replace $v_{2}$ with $w \in \mathbb{R}^{2}$ so that the particles initially located at the same positions with respective masses $m_{1}, m_{2}$ and velocities $v_{1}, w$ do not collide. Moreover, our choice in $w$ can be made so that $v_{2}$ and $w$ are as close as we desire; see Figure $6 \mathrm{~b}$. As $w$ tends to $v_{2}$, the nonintersecting trajectories in $\mathbb{R}^{2} \times[0, \infty)$ associated with the particles in Figure $6 \mathrm{~b}$ tend to two lines that intersect and not the piecewise linear trajectories that describe the perfectly inelastic collision in Figure 6a.

This type of sensitivity to small changes in initial conditions poses a challenge to understanding the evolution of the mass distribution and velocity of a collection of particles in space that interact primarily through inelastic collisions. Specifically, it prevents us from using the approximation method we discussed for $d=1$ to construct a solution pair $\rho$ and $v$ of the conservation of mass equation

$$
\partial_{t} \rho+\nabla \cdot(\rho \nu)=0
$$

and the conservation of momentum equation

$$
\partial_{t}(\rho v)+\nabla \cdot(\rho v \otimes v)=0
$$

in $\mathbb{R}^{d} \times(0, \infty)$ for a given initial mass distribution $\left.\rho\right|_{t=0}=$ $\rho_{0}$ and velocity $\left.v\right|_{t=0}=v_{0}$ when $d>1$. While there have been a few notable works on this higher dimensional initial value problem $[3,14]$, we believe this topic is still primed for mathematical innovation. Of course we hope that any new ideas which arise in the study of sticky particles dynamics in space will also provide us with a better grasp on Zel'dovich's model for the origin of galaxies. 


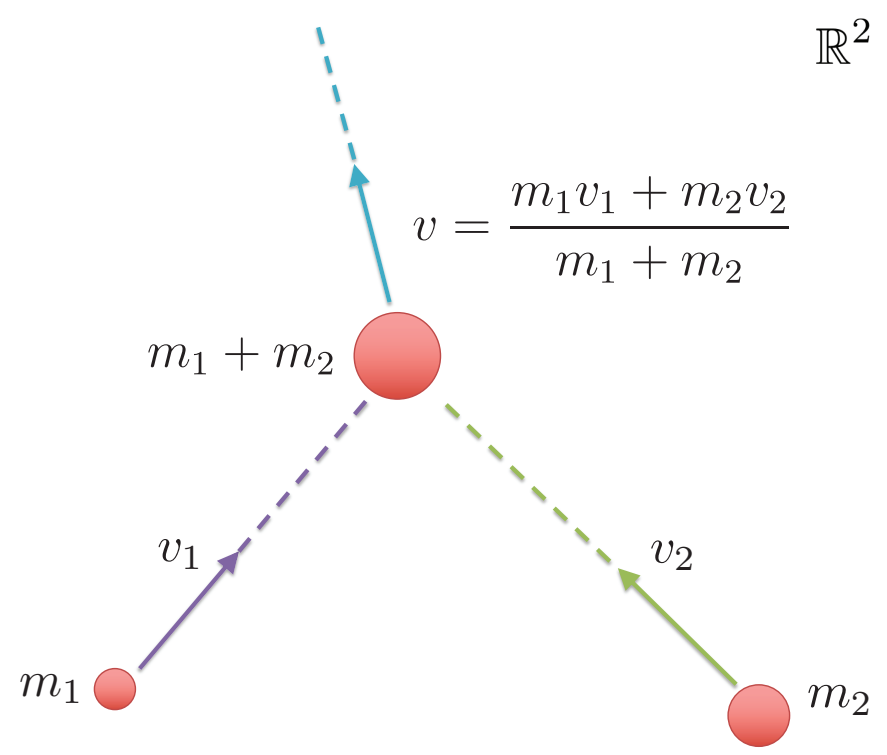

(a)

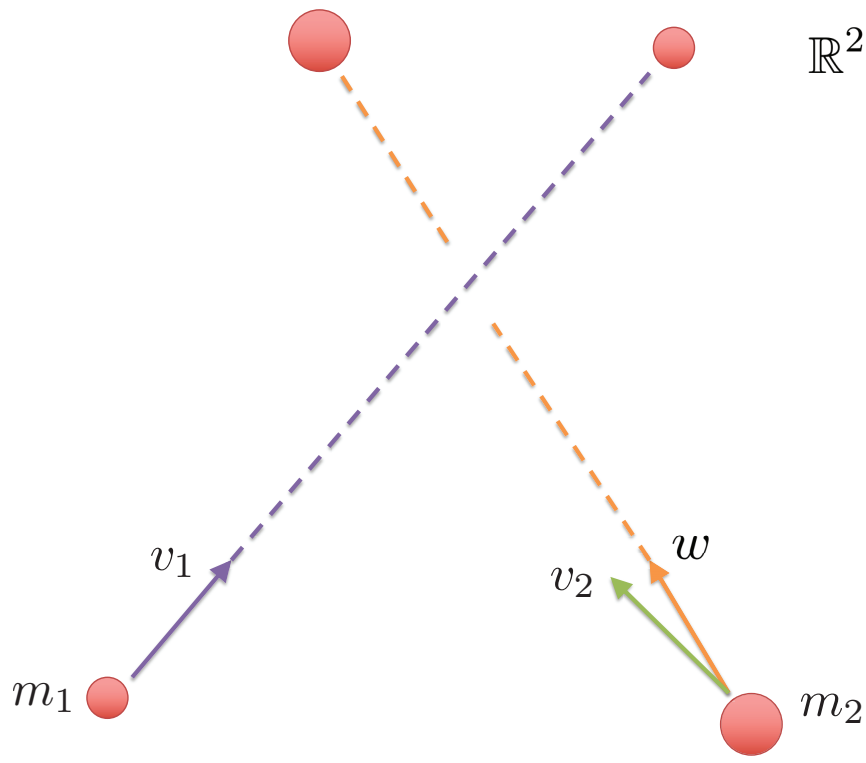

(b)

Figure 6. (a) A perfectly inelastic collision between two particles in the plane with masses $m_{1}, m_{2}$ and initial velocities $v_{1}, v_{2}$, respectively. The velocities are indicated with arrows and the corresponding paths are indicated with dashed line segments. (b) An initial velocity $w \neq v_{2}$ for the particle with mass $m_{2}$ which is selected so that the particles do not collide.

\section{References}

[1] Benamou J-D, Brenier Y, A computational fluid mechanics solution to the Monge-Kantorovich mass transfer problem. Numer. Math., 84(3):375-393, 2000. MR1738163

[2] Brenier Y, Grenier E, Sticky particles and scalar conservation laws. SIAM J. Numer. Anal., 35(6):2317-2328, 1998. MR1655848

[3] Bressan A, Nguyen T, Non-existence and non-uniqueness for multidimensional sticky particle systems. Kinet. Relat. Models, 7(2):205-218, 2014. MR3195073

[4] Dermoune A, Probabilistic interpretation of sticky particle model. Ann. Probab., 27(3):1357-1367, 1999. MR1733152

[5] Weinan E, Rykov Yu. G, Sinai Ya. G, Generalized variational principles, global weak solutions and behavior with random initial data for systems of conservation laws arising in adhesion particle dynamics. Comm. Math. Phys., 177(2):349-380, 1996. MR1384139

[6] Gangbo W, Nguyen T, Tudorascu A, Euler-Poisson systems as action-minimizing paths in the Wasserstein space. Arch. Ration. Mech. Anal., 192(3):419-452, 2009. MR2505360

[7] Gurbatov S N, Saichev A I, Shandarin S F, Large-scale structure of the universe. The Zeldovich approximation and the adhesion model. Physics-Uspekhi, 55(3):223, 2012.

[8] Huang F, Wang Z, Well posedness for pressureless flow. Comm. Math. Phys., 222(1):117-146, 2001. MR1853866

[9] Hynd R, Sticky particles and the pressureless Euler equations in one spatial dimension. arXiv preprint, 2018.

[10] Kofman L, Pogosian D, Shandarin S, Structure of the universe in the two-dimensional model of adhesion. Monthly Notices of the Royal Astronomical Society, 242(2):200-208, 1990.

[11] McCann R J, A convexity principle for interacting gases. Adv. Math., 128(1):153-179, 1997. MR1451422

[12] Monge G, Mémoire sur la théorie des déblais et de remblais. Histoire de l'Académie Royale des Sciences de Paris, avec les Mémoires de Mathématique et de Physique pour la même année, pages 666-704, 1781.

[13] Natile L, Savaré G, A Wasserstein approach to the onedimensional sticky particle system. SIAM J. Math. Anal., 41(4):1340-1365, 2009. MR2540269

[14] Sever M, An existence theorem in the large for zeropressure gas dynamics. Differential Integral Equations, 14(9):1077-1092, 2001. MR1852872

[15] Shandarin S F, Zel'dovich Ya. B, The large-scale structure of the universe: turbulence, intermittency, structures in a self-gravitating medium. Rev. Modern Phys., 61(2):185-220, 1989. MR989562

[16] Vergassola M, Dubrulle B, Frisch U, Noullez A, Burgers' equation, Devil's staircases and the mass distribution for large-scale structures. Astronomy and Astrophysics, 289:325356, 1994.

[17] Villani C, Topics in optimal transportation, volume 58 of Graduate Studies in Mathematics. American Mathematical Society, Providence, RI, 2003. MR1964483

[18] Zel'dovich Ya. B, Gravitational instability: an approximate theory for large density perturbations. Astron. Astrophys., 5:84-89, 1970. 


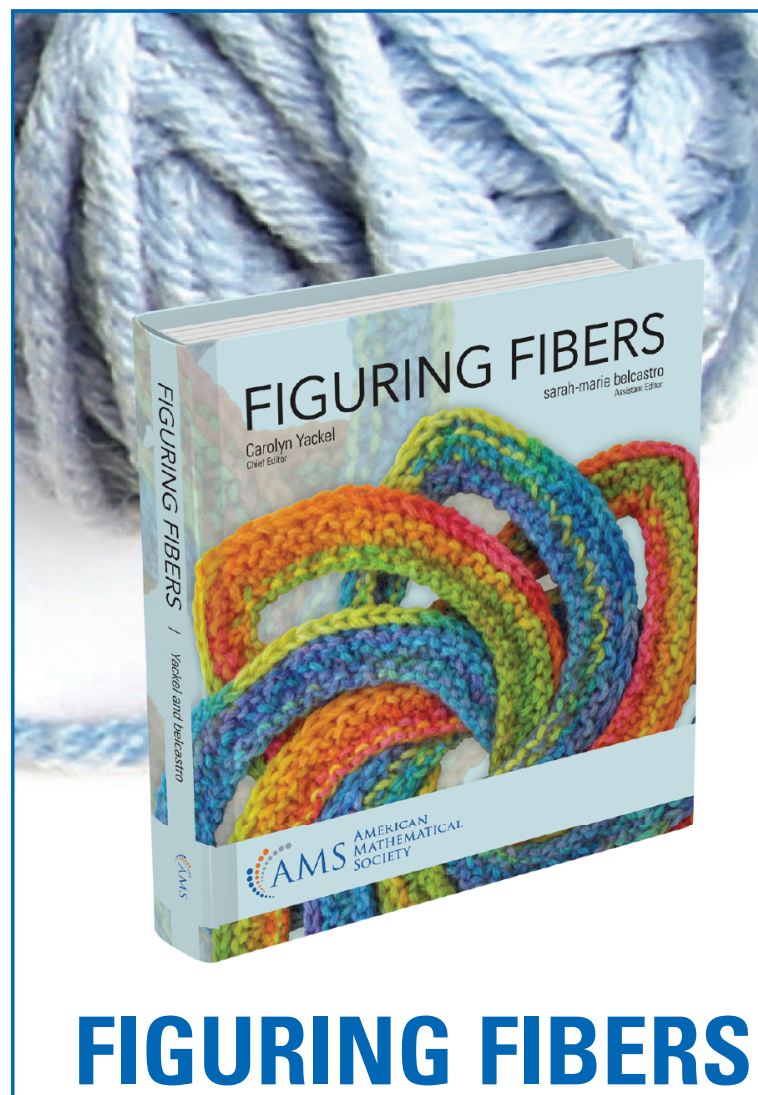

Carolyn Yackel, Mercer University, Macon, GA, Chief Editor, and sarah-marie belcastro, MathILy, Mathematical Staircase, Inc., Holyoke, $M A$, and Smith College, Northampton, MA, Assistant Editor

This book transports the reader on a mathematical exploration of fiber arts that is rigorous enough to capture the hearts of mathematicians.

Individual chapters are also available for purchase. Each self-contained chapter contains directions for a complete project in downloadable PDF format.

2018; 232 pages; Hardcover; ISBN: 978-1-4704-29317; List US\$40; AMS members US\$32; MAA members US\$36; Order code MBK/117

\section{Available at: bookstore.ams.org/figuring-fibers

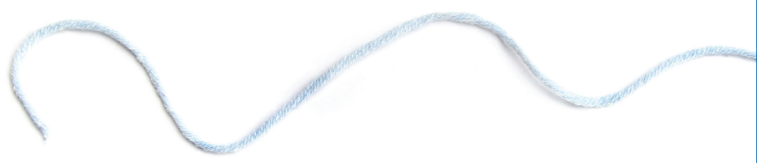

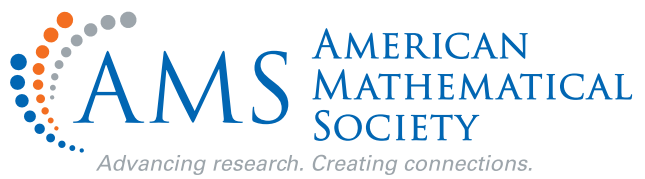

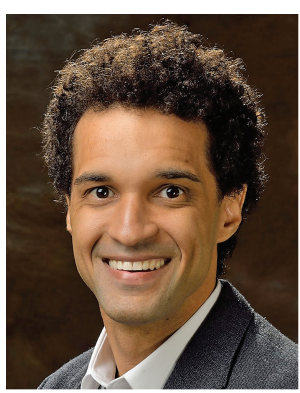

Ryan Hynd

Credits

The author photo was provided by Felice Macera. 